University of Windsor

Scholarship at UWindsor

Physics Publications

Department of Physics

1989

\title{
Eigenvalues and retardation effects in the $n=10$ states of helium
}

Gordon W. F. Drake

University of Windsor

Follow this and additional works at: https://scholar.uwindsor.ca/physicspub

Part of the Physics Commons

\section{Recommended Citation}

Drake, Gordon W. F.. (1989). Eigenvalues and retardation effects in the $n=10$ states of helium. Journal of Physics B: Atomic, Molecular and Optical Physics, 22 (23), L651-L657.

https://scholar.uwindsor.ca/physicspub/58

This Article is brought to you for free and open access by the Department of Physics at Scholarship at UWindsor. It has been accepted for inclusion in Physics Publications by an authorized administrator of Scholarship at UWindsor. For more information, please contact scholarship@uwindsor.ca. 
Eigenvalues and retardation effects in the $n=10$ states of helium

This article has been downloaded from IOPscience. Please scroll down to see the full text article.

1989 J. Phys. B: At. Mol. Opt. Phys. 22 L651

(http://iopscience.iop.org/0953-4075/22/23/001)

View the table of contents for this issue, or go to the journal homepage for more

Download details:

IP Address: 137.207.184.30

The article was downloaded on 08/05/2013 at 19:44

Please note that terms and conditions apply. 


\title{
LETTER TO THE EDITOR
}

\section{Eigenvalues and retardation effects in the $n=10$ states of helium}

\author{
G W F Drake \\ Department of Physics, University of Windsor, Windsor, Ontario, Canada N9B 3P4
}

Received 4 September 1989

\begin{abstract}
High-precision variational eigenvalues are obtained for the 10D, 10F, 10G and $10 \mathrm{H}$ states of helium by the application of multiple basis set methods. The accuracy is sufficient to provide the first definitive test of asymptotic expansion methods extensively developed by Drachman for Rydberg states of high angular momentum. The results are also compared with recent high-precision measurements for the $10 \mathrm{G}-10 \mathrm{H}$ and $10 \mathrm{~F}-10 \mathrm{G}$ transition frequencies, and interpreted in terms of predicted long-range retardation corrections. Bethe logarithms are calculated and Lamb shift corrections included. Small residual discrepancies persist which could be explained by uncalculated radiative shifts.
\end{abstract}

The suggestion by Kelsey and Spruch (1978) that Casimir-Polder retardation effects might be observable as energy shifts in the Rydberg states of helium has led to a concerted effort to observe the shifts (Palfrey et al 1984, Hessels et al 1987, 1990), particularly in the $n=10$ manifold of states. A parallel development of theory (Au et al 1984, Babb and Spruch 1988, Au 1989) provides accurate predictions for the shifts. However, retardation effects are revealed only to the extent that all the ordinary (non-retarded) effects of comparable size are known and can be subtracted from the observations. The most accurate available calculations for the fine-structure splittings in the $n=10$ states of helium are the asymptotic calculations of Drachman (1982, 1985, 1988) derived from a core polarisation model. His expansions become increasingly accurate with increasing angular momentum $L$, but contain uncertainties larger than the retardation shift for $L \leqslant 5$ where the shift is large enough to be readily observable. High-precision calculations are available for low-lying states, but a rapid loss of accuracy with increasing $n$ has until now prevented the direct application of variational methods to Rydberg states.

The purpose of this letter is to report on the application of recently developed variational techniques (Drake 1987, 1988, Drake and Makowski 1988) to the 10D, 10F, $10 \mathrm{G}$ and $10 \mathrm{H}$ states of helium. These are the first variational calculations for any Rydberg states as high as $n=10$, and the first for $G$ and $H$ states ( $L=4$ and 5 ). Convergence of the total non-relativistic energies for the $10 \mathrm{H}$ states to a few parts in $10^{17}$ makes these the most accurately determined two-electron states. The results allow retardation effects to be extracted from the experimental data to the full extent of the experimental precision. They also allow a precise assessment of the accuracy of asymptotic expansion methods.

The principal features of the calculation are as follows. The solutions to the non-relativistic two-electron Schrödinger equation are expanded in a basis set of 
Hylleraas-type functions of the form

$$
a_{i, j, k}^{(t)} r_{1}^{i} r_{2}^{j} r_{12}^{k} \exp \left(-\alpha_{t} r_{1}-\beta_{t} r_{2}\right)\left(l_{1}^{(t)}, l_{2}^{(t)} ; L\right) \pm \text { exchange }
$$

where $r_{12}=\left|r_{1}-r_{2}\right|$, the $a_{i, j, k}^{(t)}$ are linear variational coefficients and $\left(l_{1}^{(t)}, l_{2}^{(t)}, L\right)$ denotes a vector-coupled product of solid spherical harmonics with angular momenta $l_{1}^{(t)}$ and $l_{2}^{(t)}$ for the two electrons to form a state with total angular momentum $L$. The values of $l_{1}^{(t)}$ and $l_{2}^{(t)}$ required for completeness of the basis set are

$$
\left(l_{1}^{(t)}, l_{2}^{(r)}\right)=(0, L),(1, L-1), \ldots([L / 2], L-[L / 2])
$$

for $t=1,2, \ldots[L / 2]+1$, where [ ] denotes 'greatest integer in'. The novel features of the calculation which lead to a dramatic improvement in accuracy, especially for Rydberg states, are (i) the screened hydrogenic function $1 \mathrm{~s}\left(Z, r_{1}\right) n L\left(Z-1, r_{2}\right)$, where $Z$ is the nuclear charge, is included explicitly in the basis set; (ii) the terms with $(0, L)$ angular symmetry are 'doubled' in that each combination of powers $i, j, k$ in (1) is included twice with different non-linear parameters $\alpha_{t}, \beta_{t}$; and (iii) a complete optimisation of all the $\alpha_{t}, \beta_{t}$ is performed by calculating analytically the derivatives $\partial E / \partial \alpha_{t}$ and $\partial E / \partial \beta_{t}$, and locating the zeros. One set of $(0, L)$ terms represents the asymptotic behaviour of the wavefunction $\left(\alpha_{1} \simeq 2, \beta_{1} \simeq 1 / n\right)$, and the other the inner correlation effects. To these are added a further set with the same $\alpha_{1}, \beta_{1}$ and powers as the asymptotic $(0, L)$ set, but with $(1, L-1)$ angular symmetry. Without these terms, convergence becomes poor for large basis sets when the mass polarisation operator is included in the Hamiltonian. To summarise, the basis set contains the terms

$$
(0, L)_{A},(1, L-1)_{A},(0, L)_{B},(1, L-1)_{C}, \ldots([L / 2], L-[L / 2])_{X}
$$

with the first two angular sets having identical non-linear parameters. Except for the truncations described below, all combinations of powers are included in (1) such that $i+j+k \leqslant N$, and the convergence studied as $N$ is progressively increased. The truncations are $i \leqslant 3$ and $k \leqslant 2$ in set $A$, and $i+j+k+|i-j| \leqslant N$ for $k \geqslant 2$ in sets $B$ to $X$. These were carefully checked to ensure that they did not affect the convergence of the eigenvalues to within the final accuracy quoted. The truncation for sets $B$ to $X$, first suggested by Kono and Hattori (1986), only alters the order in which terms are added as $N$ increases, and so does not disrupt the ultimate completeness of the basis set. The largest basis sets contain $790,732,733$ and 785 terms, corresponding to an $N_{\max }$ of $12,11,10$ and 10 for the $D, F, G$ and $H$ states respectively.

The final non-relativistic eigenvalues, obtained by extrapolating successive differences as $N$ increases, are listed in table 1 . Since the estimated uncertainties are the ehtire amount of the extrapolation, the variational bound corresponding to the largest $N_{\max }$ basis set can be recovered by adding the uncertainty to the tabulated eigenvalue. To obtain mass polarisation corrections, all calculations were repeated with the $(\mu / \boldsymbol{M}) \boldsymbol{p}_{1} \cdot \boldsymbol{p}_{2}$ mass polarisation operator included explicitly in the Hamiltonian and the coefficient $E_{M}^{(2)}$ in the expansion

$$
E_{M}=\left[E_{\infty}+(\mu / M)\left\langle p_{1} \cdot p_{2}\right\rangle+(\mu / M)^{2} E_{M}^{(2)}\right] 2 R_{M}
$$

determined by differencing. Here, $R_{M}=(1-\mu / M) R_{\infty}$ is the reduced mass Rydberg and $\mu=M m_{\mathrm{e}} /\left(M+m_{\mathrm{e}}\right)$ is the reduced mass of the electron.

A very useful and sensitive test of the accuracy is provided by comparing the spin-averaged $\bar{E}_{M}^{(2)}=\frac{1}{2}\left[E_{M}^{(2)}\left({ }^{1} L\right)+E_{M}^{(2)}\left({ }^{3} L\right)\right]$ as obtained above from the variational 
Table 1. Non-relativistic eigenvalues for the $10 \mathrm{D}, 10 \mathrm{~F}, 10 \mathrm{G}$ and $10 \mathrm{H}$ states of ${ }^{4} \mathrm{He}$, expressed as a correction $\Delta E_{\infty}$ to the screened hydrogenic energy $E_{\mathrm{SH}_{\mathrm{H}}}=-2.005 \mathrm{au}$. The $\Delta E_{M}$ results include the mass polarisation operator in the Hamiltonian $H=H_{0}+(\mu / M) \boldsymbol{p}_{1} \cdot \boldsymbol{p}_{2}$ with $\mu / M=1.370745620 \times 10^{-4}$. The last column gives matrix elements of $\pi \delta\left(\boldsymbol{r}_{1}\right)$ for finite nuclear mass.

\begin{tabular}{lccc}
\hline State & $\Delta E_{\infty}\left(10^{-8} \mathrm{au}\right)$ & $\Delta E_{M}\left(10^{-8} \mathrm{au}\right)$ & $\pi\left\langle\delta\left(\boldsymbol{r}_{1}\right)\right\rangle-4\left(10^{-6} \mathrm{au}\right)^{\mathrm{a}}$ \\
\hline $10^{1} \mathrm{D}$ & $-207.1654245(1)^{\mathrm{b}}$ & $-207.3113099(5)$ & -13.87 \\
$10^{3} \mathrm{D}$ & $-281.8080224(1)$ & $-281.7734965(5)$ & -18.58 \\
$10^{\mathrm{l}} \mathrm{F}$ & $-41.7564669(2)$ & $-41.7769603(1)$ & -2.846 \\
$10^{3} \mathrm{~F}$ & $-42.1686604(1)$ & $-42.1883255(1)$ & -2.882 \\
$10^{1} \mathrm{G}$ & $-11.276431780(2)$ & $-11.288856644(8)$ & -0.7740 \\
$10^{3} \mathrm{G}$ & $-11.277700278(4)$ & $-11.290122434(6)$ & -0.7741 \\
$10^{1} \mathrm{H}$ & $-3.921439451(1)$ & $-3.931901064(1)$ & -0.2697 \\
$10^{3} \mathrm{H}$ & $-3.921441740(1)$ & $-3.931903346(1)$ & -0.2697 \\
\hline
\end{tabular}

${ }^{a}$ For large $L, \pi\left\langle\delta\left(r_{1}\right)\right\rangle$ tends asymptotically to $4-\frac{31}{32}\left\langle x^{-4}\right\rangle+\mathrm{O}\left(x^{-6}\right)$ au.

${ }^{b}$ Numbers in brackets denote the downward extrapolation contained in the final figure quoted.

eigenvalues with $\bar{E}_{\mathrm{pol}}^{(2)}$ calculated from the polarisation model of Drachman (1988). The values for the $10 \mathrm{H}$ state are (in atomic units)

$$
\begin{aligned}
\bar{E}_{\mathrm{pol}}^{(2)} & =-\frac{1}{2} n^{-2}-\frac{5}{2} \alpha_{1}\left\langle x^{-4}\right\rangle-\left(4 \alpha_{2}-15 \beta_{1}\right)\left\langle x^{-6}\right\rangle+\mathrm{O}\left(x^{-7}\right) \\
& =-0.005000196
\end{aligned}
$$

and

$$
\bar{E}_{M}^{(2)}=-0.005000194
$$

where $\alpha_{L}$ is the $2^{L}$-pole polarisability of the $\mathrm{He}^{+}(1 \mathrm{~s})$ core, $\beta_{1}$ is the leading non-adiabatic correction, and the expectation values are with respect to the screened hydrogenic wavefunction of the outer electron. The difference $(\mu / M)^{2}\left(\bar{E}_{M}^{(2)}-\bar{E}_{\text {pol }}^{(2)}\right) \simeq 4 \times 10^{-17}$ au $(0.3 \mathrm{~Hz})$ is the same order of magnitude as the estimated uncertainty in the extrapolated eigenvalues for the $10 \mathrm{H}$ states.

Of more direct importance is the comparison with Drachman's polarisation results in table 2 for the corrections to the screened hydrogenic energy

$$
E_{\mathrm{SH}}=\left(-2-\frac{1}{2} n^{-2}\right) 2 R_{M}
$$

In his notation, $V_{i}$ is the contribution to the energy from terms of order $x^{-i}$ in the long-range interaction. The quantity tabulated for $\mathrm{G}$ and $\mathrm{H}$ states corresponds to Drachman (1985) $V_{4}+V_{6}+\frac{1}{2}\left(V_{7}+V_{8}\right)+\Delta_{2} \pm \frac{1}{2}\left(V_{7}+V_{8}\right)$, where $\Delta_{2}$ is the second-order dipole correction. For $F$ states, it is $V_{4}+\frac{1}{2} V_{6}+\Delta_{2} \pm \frac{1}{2} V_{6}$. The spectacularly good agreement for $10 \mathrm{G}$ and $10 \mathrm{H}$ is a somewhat fortuitous consequence of including only

Table 2. Comparison of the spin-averaged variational eigenvalues $\Delta \bar{E}_{\mathrm{var}}$ with the corresponding $\Delta \vec{E}_{\text {pol }}$ obtained from Drachman's asumptotic expansion (see text).

\begin{tabular}{llcc}
\hline State & $\Delta \bar{E}_{\text {var }}(\mathrm{MHz})^{\mathrm{a}}$ & $\Delta \bar{E}_{\mathrm{poj}}(\mathrm{MHz})$ & Difference \\
\hline $10 \mathrm{~F}$ & -2760.626 & $-2778.708 \pm 45.042$ & 18.082 \\
$10 \mathrm{G}$ & -741.8936 & $-741.8956 \pm 0.5207$ & 0.0020 \\
$10 \mathrm{H}$ & -257.9830 & $-257.9817 \pm 0.0097$ & -0.0013 \\
\hline
\end{tabular}

a The value of the Rydberg used is $R_{M}=3289391007 \mathrm{MHz}$. 
$\frac{1}{2}\left(V_{7}+V_{8}\right)$ in the sum and regarding the remaining $\frac{1}{2}\left(V_{7}+V_{8}\right)$ as the uncertainty. It remains to be seen if this prescription works as well in other cases.

The main point of this letter is to compare the total transition energies with the recent high precision measurements of Hessels et al (1990). Table 3 summarises the contributions to the energy for the $10 \mathrm{D}, 10 \mathrm{~F}, 10 \mathrm{G}$ and $10 \mathrm{H}$ states. The entries in the table are as follows. $\Delta E_{\mathrm{nr}}$ is the correction to the screened hydrogenic energy $E_{\mathrm{SH}}$, $\Delta E_{M}^{(1)}$ and $\Delta E_{M}^{(2)}$ are the first- and second-order mass polarisation corrections, $\Delta E_{\text {rel }}$ is the relativistic correction, $\Delta E_{\text {anom }}$ is the anomalous magnetic moment correction, $\Delta E_{\text {st }}$ is the singlet-triplet mixing correction, $\left(\Delta E_{\mathrm{RR}}\right)_{M}$ is the relativistic reduced mass correction from the mass scaling of the Breit interaction together with the Stone (1963) terms, $\left(\Delta E_{\mathrm{RR}}\right)_{X}$ is a second order cross term between the Breit interaction and the mass polarisation operator, and $\Delta E_{1,1}$ and $\Delta E_{\mathrm{L}, 2}$ are one- and two-electron Lamb shift corrections. Detailed expressions for all of these terms have been given previously (Drake and Makowski 1988), and will not be repeated here. All are expressed relative to the $\mathrm{He}^{+}(1 \mathrm{~s})$ state. The one change from our previous work involves the evaluation of the two-electron Bethe logarithm in $\Delta E_{\mathrm{L}, 1}$, as discussed below. The Bethe logarithms needed for the $n=10$ states are listed in table 4 . These were obtained by a direct summation method analogous to that used by Klarsfeld and Maquet (1973). The hydrogen 1s value agrees to the figures quoted with the calculation of Haywood and Morgan (1985).

An important conclusion from table 3 is that the $\left(\Delta E_{\mathrm{RR}}\right)_{M}$ and $\left(\Delta E_{\mathrm{RR}}\right)_{X}$ terms nearly cancel for the higher values of $L$. It would therefore be better to omit relativistic reduced mass corrections altogether (other than the elementary correction from $R_{M}$ ), rather than including them only partially in an approximate calculation.

Table 5 compares the calculated F-G and G-H transition frequencies with the measured values. For the $\mathrm{G}-\mathrm{H}$ transitions, the fine-structure and singlet-triplet splitting within each manifold of states is reproduced to within the $\pm 2 \mathrm{kHz}$ accuracy of the measurements, but there is a systematic discrepancy of $5 \pm 2 \mathrm{kHz}$ for all the transitions. A similar discrepancy does not appear to be present for the F-G transitions.

The significance of these results for the theory of retardation corrections is as follows. As discussed by $\mathrm{Au}$ et al (1984) and Au (1988), the retardation terms for $x \leqslant 137 a_{0}$ are (in atomic units)

$$
\Delta V=\frac{\alpha^{2}}{4}\left(\frac{a_{0}}{x}\right)^{4}-\frac{7 \alpha^{3}}{6 \pi}\left(\frac{a_{0}}{x}\right)^{3}+\mathrm{O}\left[\alpha^{4}\left(a_{0} / x\right)^{2}\right] .
$$

The first term above is a long-range approximation to the orbit-orbit $\left(\mathrm{H}_{2}\right)$ term in the Breit interaction, and so is automaically included in $\Delta E_{\text {rel }}$. The second term is a long range approximation to

$$
\Delta E_{\mathrm{L}, 2}=\alpha^{3}\left(\frac{14}{3} \ln \alpha+\frac{164}{15}\right)\left\langle\delta\left(\boldsymbol{r}_{12}\right)\right\rangle-\frac{14}{3} \alpha^{3} Q
$$

with

$$
Q=\frac{1}{4 \pi} \lim _{a \rightarrow 0}\left\langle r_{12}^{-3}(a)+4 \pi(\gamma+\ln a) \delta\left(r_{12}\right)\right\rangle
$$

in which $\left\langle\delta\left(\boldsymbol{r}_{12}\right)\right\rangle$ is neglected and $r_{12}^{-3}$ is approximated by $x^{-3}$. (Here $a$ is the radius of a small sphere about $r_{12}=0$ which is excluded from the integration.) This term is therefore also automatically included in the total energy. For the G-H and F-G transitions, the contribution from the two terms in (6) is $-41.49 \mathrm{kHz}$ and $-184.1 \mathrm{kHz}$ respectively. without these terms, theory and experiment would seriously disagree. What is not included in the calculation are the slight differences between (6) and the 


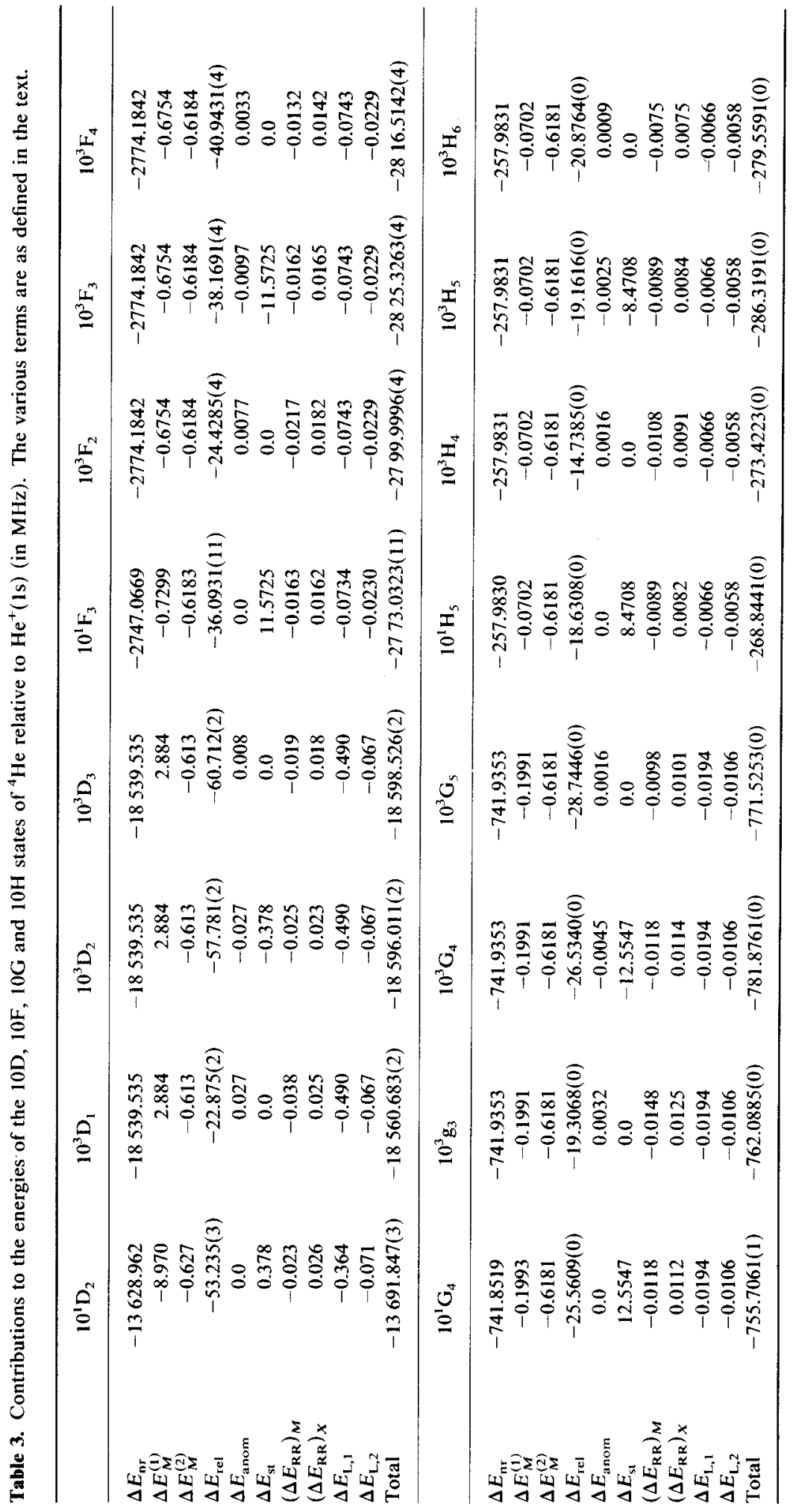


Table 4. Two-electron Bethe logarithms for the $n=10$ states of helium, as calculated from equation (10). The second column gives the corresponding Bethe logarithm for hydrogen, correct to the figures quoted.

\begin{tabular}{lll}
\hline State & $\ln \left(k_{0}(n l) / R_{\infty}\right)$ & $\ln \left(k_{0}(1 s n l) / Z^{2} R_{\infty}\right)$ \\
\hline $1 \mathrm{~S}$ & 2.9841285557655 & \\
$10 \mathrm{~S}$ & 2.7276469386595 & 2.9813177159832 \\
$10 \mathrm{P}$ & -0.0474828933567 & 2.9841255880847 \\
$10 \mathrm{D}$ & -0.0091322722490 & 2.9841279849985 \\
$10 \mathrm{~F}$ & -0.0030590942789 & 2.9841283645721 \\
$10 \mathrm{G}$ & -0.0013197180574 & 2.9841284732831 \\
$10 \mathrm{H}$ & -0.0006568860162 & 2.9841285147101 \\
$10 \mathrm{I}$ & -0.0003572986495 & 2.9841285334343 \\
$10 \mathrm{~K}$ & -0.0002055849884 & 2.9841285429164 \\
$10 \mathrm{~L}$ & -0.0001222846308 & 2.9841285481227 \\
$10 \mathrm{M}$ & -0.0000737249786 & 2.9841285511577 \\
\hline
\end{tabular}

Table 5. Comparison of theory and experiment for the $10 \mathrm{~F}-10 \mathrm{G}$ and $10 \mathrm{G}-10 \mathrm{H}$ transition frequencies of ${ }^{4} \mathrm{He}$ (in $\mathrm{MHz}$ ).

\begin{tabular}{llll}
\hline Transition & Experiment & Theory & Difference \\
\hline${ }^{1} \mathrm{~F}_{3}-{ }^{1} \mathrm{G}_{4}$ & $2017.325(3)$ & 2017.326 & $-0.001(3)$ \\
${ }^{3} \mathrm{~F}_{2}-{ }^{3} \mathrm{G}_{3}$ & $2037.910(5)$ & 2037.911 & $-0.001(3)$ \\
${ }^{3} \mathrm{~F}_{3}-{ }^{3} \mathrm{G}_{4}$ & $2043.452(5)$ & 2043.450 & $0.002(5)$ \\
${ }^{3} \mathrm{~F}_{4}-{ }^{3} \mathrm{G}_{4}$ & $2044.984(4)$ & 2044.989 & $-0.005(4)$ \\
${ }^{1} \mathrm{G}_{4}-{ }^{1} \mathrm{H}_{5}$ & $486.866(2)$ & 486.8620 & $0.004(2)$ \\
${ }^{3} \mathrm{G}_{3}-{ }^{3} \mathrm{H}_{4}$ & $488.672(2)$ & 488.6662 & $0.006(2)$ \\
${ }^{3} \mathrm{G}_{4-}{ }^{3} \mathrm{H}_{5}$ & $495.561(2)$ & 495.5570 & $0.004(2)$ \\
${ }^{3} \mathrm{G}_{5}-{ }^{3} \mathrm{H}_{6}$ & $491.971(2)$ & 491.9662 & $0.005(2)$ \\
\hline
\end{tabular}

${ }^{a}$ Hessels et al (1990). Numbers in brackets denote the experimental uncertainty in the final figure quoted.

fully retarded values calculated by Babb and Spruch (1988). For the G-H transition, they obtain $20.486-62.686=-42.20 \mathrm{kHz}$. The $-0.71 \mathrm{kHz}$ difference between this and the result of (6) is much less than the $5 \pm 2 \mathrm{kHz}$ difference between theory and experiment, and so cannot account for the discrepancy. The $-0.71 \mathrm{kHz}$ difference reflects the part of the long-range Casimir-Polder retardation effect which is not included as part of the usual terms evaluated for low-lying states.

What has apparently not been appreciated before is that the one-electron Lamb shift enters at the $\pm 20 \mathrm{kHz}$ level of accuracy for the G-H transition. The leading terms in the quantity tabulated in table 3 are

$$
\begin{aligned}
& \Delta E_{\mathrm{L}, 1}=\frac{4}{3} Z \alpha^{3}\langle\left.\delta\left(\boldsymbol{r}_{1}\right)+\delta\left(\boldsymbol{r}_{2}\right)\right\rangle\left[\ln (Z \alpha)^{-2}-\ln \left(k_{0}(1 \mathrm{~s}, n l) / Z^{2} R_{\infty}\right)+\frac{19}{30}+\mathrm{O}(\alpha Z)\right\} \\
&-\frac{4}{3} Z \alpha^{3}\left(Z^{3} / \pi\right)\left[\ln (Z \alpha)^{-2}-\ln \left(k_{0}(1 \mathrm{~s}) / Z^{2} R_{\infty}\right)+\frac{19}{30}+\mathrm{O}(\alpha Z)\right] .
\end{aligned}
$$

where the second term subtracts the corresponding $\mathrm{He}^{+}(1 \mathrm{~s})$ Lamb shift. The main contributions come from the departure of $\left\langle\delta\left(\boldsymbol{r}_{1}\right)+\delta\left(\boldsymbol{r}_{2}\right)\right\rangle$ from the hydrogenic value $Z^{3} / \pi$ (see table 1 ) and the departure of the two-electron Bethe logarithm $\ln \left[k_{0}(1 \mathrm{~s}, n l)\right]$ from the hydrogenic value. The two effects act in opposite directions. To lowest order 
in a $Z^{-1}$ expansion, the two-electron Bethe logarithm is (Ermolaev and Swainson 1983, Goldman and Drake 1984)

$$
\ln \left(\frac{k_{0}(1 \mathrm{~s} n l, Z)}{Z^{2} R_{\infty}}\right)=\ln \left(\frac{k_{0}(1 \mathrm{~s})}{R_{\infty}}\right)+n^{-3} \ln \left(\frac{k_{0}(n l)}{R_{\infty}}\right)
$$

for $l \neq 0$, where the $k_{0}(n l)$ denote hydrogen atom Bethe logarithms. However, for Rydberg states, the above should be modified to read

$$
\ln \left(\frac{k_{0}(1 \mathrm{~s} n l, Z)}{Z^{2} R_{\infty}}\right)=\ln \left(\frac{k_{0}(1 \mathrm{~s})}{R_{\infty}}\right)+\frac{1}{n^{3}}\left(\frac{Z-1}{Z}\right)^{4} \ln \left(\frac{k_{0}(n l)}{R_{\infty}}\right)
$$

corresponding to the physical picture in which one calculates the mean excitation energy of the $1 \mathrm{~s}$ electron for an unscreened nuclear charge of $Z$, and the $n l$ electron for a screened nuclear charge of $Z-1$. without this modification, the discrepancies between theory and experiment become $15 \mathrm{kHz}$ and $28 \mathrm{kHz}$ for the $\mathrm{G}-\mathrm{H}$ and $\mathrm{F}-\mathrm{G}$ transitions respectively, which is several times larger than the experimental uncertainty. The extra factor of $[(Z-1) / Z]^{4}$ in (10) strongly suppresses the Bethe logarithm correction to the energy relative to $\mathrm{He}^{+}(1 \mathrm{~s})$, so that the $\Delta E_{\mathrm{L}, 1}$ values listed in table 3 come primarily from the $\left\langle\delta\left(\boldsymbol{r}_{1}\right)+\delta\left(\boldsymbol{r}_{2}\right)\right\rangle$ correction multiplying the large 1s Lamb shift. For $l=0$, the right-hand side of (10) should be divided by $1+[(Z-1) / Z]^{4} / n^{3}$.

In conclusion, the results of this letter verify that the leading terms in the long-range retardation correction are in agreement with experiment to an accuracy of $\pm 10 \%$ or better. The non-relativistic eigenvalues are the most accurate reported for any twoelectron state. It is clear that further improvements in accuracy below the $\pm 1 \mathrm{kHz}$ level will require a more detailed knowledge of radiative corrections for Rydberg states.

This research was supported in part by the National Sciences and Engineering Research Council of Canada and in part by the US Department of Energy Office of Basic Energy Sciences under contract W-31-109-ENG-38. The author is grateful to the Argonne National Laboratory for its hospitality during the completion of this work, and to $\mathrm{Dr}$ $\mathrm{S} R$ Lundeen for communicating his experimental results in advance of publication.

\section{References}

Au C. K 1989 Phys. Rev. A 39 2789-95

Au C K, Feinberg G and Sucher J 1984 Phys. Rev. Lett. 53 1145-8

Babb J F and Spruch L 1988 Phys. Rev. A 38 13-8

Drachman R J 1982 Phys. Rev. A 26 1228-38

- 1985 Phys. Rev. A 31 1253-8 1988 Phys. Rev. A 37 979-80

Drake G W F 1987 Phys. Rev. Lett. 59 1549-52

- $1988 \mathrm{Nucl}$. Instrum. Methods B 31 7-13

Drake G W F and Makowski A J 1988 J. Opt. Soc. Am. B 5 2207-13

Ermolaev A M and Swainson R A 1983 J. Phys. B: At. Mol. Phys, 16 L35-42

Goldman S P and Drake G W F 1984 J. Phys. B: At. Mol. Phys. 17 L197-202

Haywood S E and Morgan J D III 1985 Phys. Rev. A 32 3179-86

Hessels E A, Deck F J, Arcuni P W and Lundeen S R 1990 Phys. Rev. A in press

Hessels E A, Sturrus W G, Lundeen S R and Cok D R 1987 Phys. Rev. A 35 4489-98

Kelsey E J and Spruch L 1978 Phys. Rev. A 18 15-25

Klarsfeld S and Maquet A 1973 Phys. Lett. 43B 201-4

Kono A and Hattori S 1986 Phys. Rev. A 34 1727-35

Palfrey S L and Lundeen S R 1984 Phys. Rev. Lett. 53 1141-4

Stone A P 1963 Proc. Phys. Soc. 81 868-76 